March 2008

Revised:

April 2008

\title{
Off resonance background effects in $e^{+} e^{-} \rightarrow t \bar{t} H$
}

\author{
Karol Kołodziej] and Szymon Szczypiński2 \\ Institute of Physics, University of Silesia \\ ul. Uniwersytecka 4, PL-40007 Katowice, Poland
}

\begin{abstract}
The top quark Yukawa coupling to the intermediate mass Higgs boson can be determined in the reaction $e^{+} e^{-} \rightarrow t \bar{t} H$ that, after taking into account decays, will be detected at the International Linear Collider through reactions with 8 particles in the final state. Such $2 \rightarrow 8$ reactions receive contributions from tens thousands of Feynman diagrams, already in the lowest order of Standard Model, most of which comprise background to resonant associated production and decay of the top quark pair and Higgs boson. We illustrate the background effects by comparing cross sections of three reactions, which represent different detection channels of $e^{+} e^{-} \rightarrow t \bar{t} H$, calculated with the complete sets of the lowest order Feynman diagrams with the corresponding signal cross sections calculated with the diagrams of associated production and decay of off mass shell top quark pair and Higgs boson only. The comparison that is performed with different selections of cuts shows that the background effects are sizeable, but they can be reduced by appropriate choice of cuts.
\end{abstract}

\footnotetext{
${ }^{1}$ E-mail: karol.kolodziej@us.edu.pl

${ }^{2}$ E-mail: simon@server.phys.us.edu.pl
} 


\section{Introduction}

If the Standard Model (SM) Higgs boson has mass below the $t \bar{t}$ threshold, $m_{H}<2 m_{t}$, then its Yukawa coupling to the top quark

$$
g_{t t H}=\frac{m_{t}}{v}, \quad \text { with } \quad v=\left(\sqrt{2} G_{F}\right)^{-1 / 2} \simeq 246 \mathrm{GeV},
$$

which is by far the largest Yukawa coupling of SM, can be best determined at the future International Linear Collider (ILC) [1] through measurement of the reaction of associated production of the top quark pair and Higgs boson [2]

$$
e^{+} e^{-} \rightarrow t \bar{t} H
$$

The lowest order SM Feynman diagrams of reaction (1), with the neglect of the Higgs boson coupling to electrons, are shown in Fig. 1. As the contribution of the Higgs boson emission off

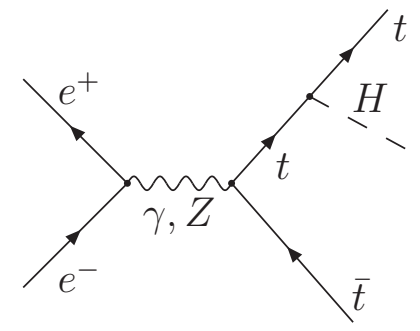

(a)

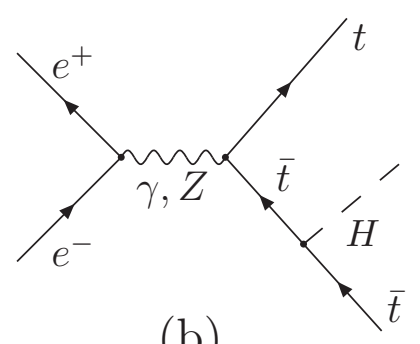

(b)

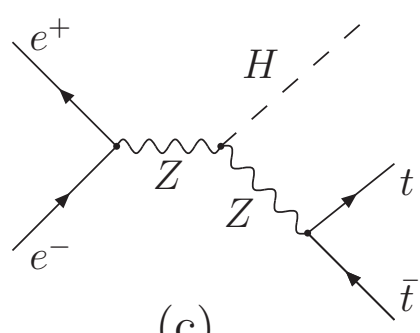

(c)

Figure 1: Feynman diagrams of reaction (11) to the lowest order of SM with the neglect of the Higgs boson coupling to electrons.

the virtual $Z$-boson line, which is represented by the diagram in Fig. 1 $k$, is small with respect to the Higgsstrahlung off the top quark line illustrated in Fig. 1a and 1 b, the SM lowest order cross section of reaction (11) becomes practically proportional to $g_{t t H}^{2}$. This fact makes reaction (11) so sensitive to the top-Higgs Yukawa coupling.

Particles on the right hand side of reaction (11) are unstable: the top and antitop decay, even before they hadronize, predominantly into $b W^{+}$and $\bar{b} W^{-}$, respectively, and the Higgs boson, dependent on its mass, decays either into a fermion-antifermion or an electroweak (EW) gauge boson pair and the EW bosons subsequently decay, each into a fermion-antifermion pair. This, dependent on the Higgs boson mass $m_{H}$, leads to reactions with either 8 or 10 fermions in the final state. As direct searches for the Higgs boson at LEP, which give a lower limit for $m_{H}$ of $114.4 \mathrm{GeV}$ at $95 \% \mathrm{CL}$ [3], combined with theoretical constrains in the framework of SM favour a value of $m_{H}$ in the range just above the lower direct search limit [4], we will assume $m_{H}<140 \mathrm{GeV}$. Then the Higgs boson would decay dominantly into a $b \bar{b}$-quark pair and reaction (1) will be actually detected at the ILC through reactions of the form

$$
e^{+} e^{-} \rightarrow b \bar{b} b \bar{b} f_{1} \bar{f}_{1}^{\prime} f_{2} \bar{f}_{2}^{\prime}
$$

where $f_{1}, f_{2}^{\prime}=\nu_{e}, \nu_{\mu}, \nu_{\tau}, u, c$ and $f_{1}^{\prime}, f_{2}=e^{-}, \mu^{-}, \tau^{-}, d, s$. Thus, reaction (2) can be detected in any of the following channels: the hadronic (38\%), leptonic (25\%), or semileptonic (37\%), 
corresponding to decay modes of the $W$-bosons coming from decays of the $t$ - and $\bar{t}$-quark of reaction (11).

Since the original work of the early 1990's [2] reaction (11) has received a lot of attention in literature. The quantum chromodynamics (QCD) radiative corrections to it were calculated in [5], $\mathcal{O}(\alpha)$ EW corrections were calculated in [6] and full $\mathcal{O}(\alpha)$ EW and $\mathcal{O}\left(\alpha_{s}\right)$ QCD corrections were studied in [7]. Reaction (11) was considered in the kinematic region where the Higgs boson energy is close to its maximal energy in 8]. Processes of the form $e^{+} e^{-} \rightarrow b \bar{b} b \bar{b} W^{+} W^{-} \rightarrow$ $b \bar{b} b \bar{b} l^{ \pm} \nu_{l} q \bar{q}^{\prime}$ accounting for the signal of associated Higgs boson and top quark pair production, as well as several irreducible background reactions, were studied in [9] and EW contributions to the leptonic and semileptonic reactions (2) have been computed in [10]. Pure off mass shell effects in $e^{+} e^{-} \rightarrow t^{*} \bar{t}^{*} H^{*} \rightarrow b \bar{b} b \bar{b} u \bar{d} \mu^{-} \bar{\nu}_{\mu}$ have been discussed in [11] and, for the on-shell Higgs boson, in [12]. In the latter, also the off resonance background contributions have been calculated. Moreover, feasibility of the measurement of the top-Higgs Yukawa coupling at the ILC in reaction (1) was discussed in [13].

Already in the lowest order of SM reactions (2) receive contributions from tens thousands of Feynman diagrams, most of which comprise background to the resonant production and decay of the top quark pair and Higgs boson. In order to show what a role the off resonance background effects will play we select one reaction in each of the different detection channels of (2): the hadronic, semileptonic and leptonic channel

$$
\begin{aligned}
& e^{+} e^{-} \rightarrow b \bar{b} b \bar{b} u \bar{d} s \bar{c}, \\
& e^{+} e^{-} \rightarrow b \bar{b} b \bar{b} u \bar{d} \mu^{-} \bar{\nu}_{\mu}, \\
& e^{+} e^{-} \rightarrow b \bar{b} b \bar{b} \tau^{+} \nu_{\tau} \mu^{-} \bar{\nu}_{\mu},
\end{aligned}
$$

respectively. We will calculate the lowest order SM cross sections of (3), (44) and (5) with the complete set of the lowest order Feynman diagrams, both EW and QCD ones, and compare them with the corresponding signal cross sections of the associated resonant production and decay of the top quark pair and Higgs boson, i.e. cross sections calculated with the signal Feynman diagrams obtained from those depicted in Fig. 1 by attaching to each final state particle of (11) lines representing its decay products. Taking into account permutations of the $b$ and $\bar{b}$ quark lines gives 20 signal diagrams for each of reactions (3), (4) and (5). For illustration, we show representative signal diagrams for (4) in Fig. 2, The comparison will be performed with different selections of cuts on particle angles, energies, missing transverse energy and invariant masses which should allow for correct identification of the signal and reduction of the background.

\section{Details of calculation}

Matrix elements of reactions (3), (44) and (5) have been generated automatically with a Fortran 90/95 program carlomat [14] written by one of us (KK). Fermion masses, except for the neutrinos, can be kept nonzero in the program, but in order to speed up the calculation we neglect masses and Yukawa couplings of the fermions lighter than $c$ quark and $\tau$ lepton. Then, taking into account both the EW and QCD lowest order contributions in the unitary gauge, there are 39342, 26816 and 21214 Feynman diagrams for (3), (4) and (5), respectively. For 


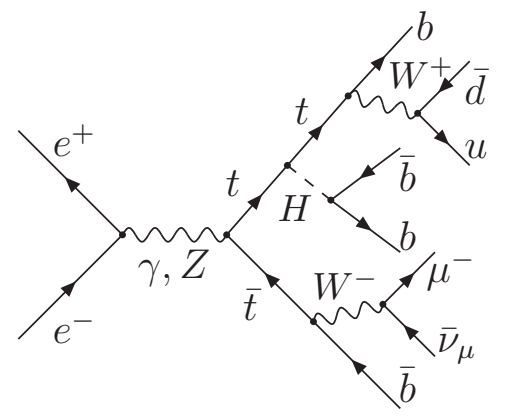

(a)

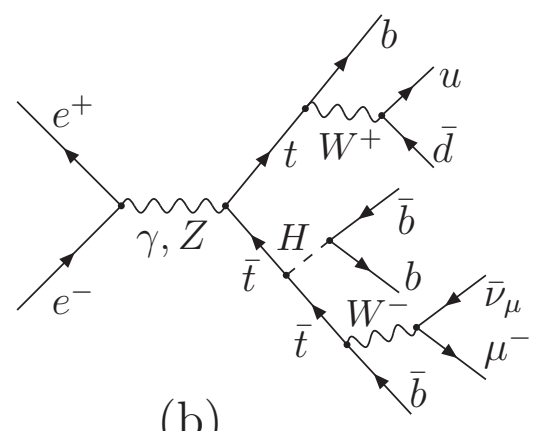

(b)

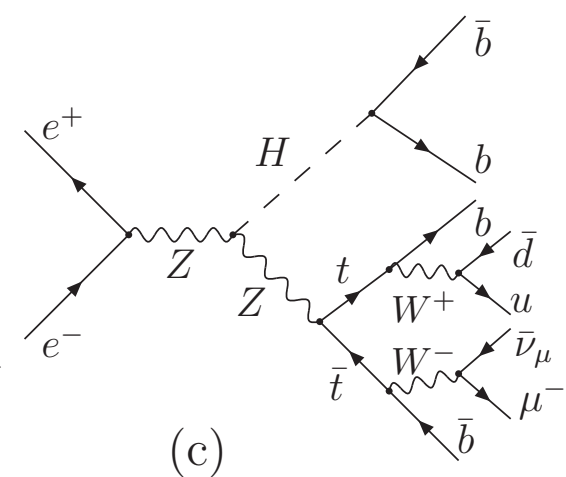

(c)

Figure 2: Representative signal Feynman diagrams of reaction (44). The remaining diagrams are obtained by all possible permutations of the two $b$ and two $\bar{b}$ lines. The Higgs boson coupling to electrons has been neglected.

each of the reactions carlomat generates also dedicated phase space parametrizations which take into account mappings of peaks in the matrix element caused by propagators of massive unstable particles, of a photon, or a gluon in each Feynman diagram. This means that a number of different phase space parametrizations generated is equal to a number of the Feynman diagrams. The phase space parametrizations are implemented into a multichannel Monte Carlo (MC) integration routine that performs integration over a 20-dimensional phase space. However, only those parametrizations which result in different phase space normalization are effectively used in the process of numerical integration.

Poles in the propagators of unstable particles are regularized with constant particle widths $\Gamma_{a}$ which are introduced through the complex mass parameters $M_{a}^{2}$ by making the substitution

$$
m_{a}^{2} \rightarrow M_{a}^{2}=m_{a}^{2}-i m_{a} \Gamma_{a}, \quad a=Z, W, H, t .
$$

Substitution (66) is made both in the $s$ - and $t$-channel propagators. The electroweak mixing parameter $\sin ^{2} \theta_{W}$ can be kept real

$$
\sin ^{2} \theta_{W}=1-\frac{m_{W}^{2}}{m_{Z}^{2}}
$$

which is the approach usually referred to in the literature as the fixed width scheme (FWS), or it may be defined as a complex quantity

$$
\sin ^{2} \theta_{W}=1-\frac{M_{W}^{2}}{M_{Z}^{2}},
$$

that is referred to as the complex mass scheme (CMS) [15]. The latter has the advantage that it preserves the lowest order Ward identities, which minimizes gauge invariance violation effects caused by (무).

The widths $\Gamma_{a}$, except for $\Gamma_{Z}$ whose actual value is rather irrelevant in the context of associated top quark pair and Higgs boson production and decay, are calculated in the lowest order of SM. In the calculation of matrix elements with the helicity amplitude method, use is made of the routines developed for a MC program eett6 $\mathrm{f} v$. 1.0, for calculating lowest order cross sections of $e^{+} e^{-} \rightarrow 6$ fermions relevant for a $t \bar{t}$ pair production and decay [16], which have been 
tailored to meet needs of the automatic generation of amplitudes in carlomat. MC summing over helicities is performed.

We have performed a number of tests of our results. First off all, we have generated the matrix element of (44) keeping the Yukawa couplings and masses of all charged fermions, which results in 56550 Feynman diagrams, and performed the phase space integration with the corresponding number of different phase space parametrizations. The results have agreed up to one standard deviation of the MC integration with those presented in the next section, which have been obtained with 26816 Feynman diagrams after neglecting the light fermion masses and the Higgs boson couplings to light fermions. Moreover, carlomat offers an option that allows to map poles caused by propagators of internal particles which decay into $2,3,4$, or more external particles. This has led to different phase space parametrizations which all have given results consistent within 1-2 standard deviations. In addition to these self-consistency checks we have compared our results for the cross sections of (4) without QCD contributions with WHIZARD/OMEGA [17] obtaining agreement within one standard deviation.

Generation of the Fortran code with carlomat on a PC with the Pentium $43.0 \mathrm{GHz}$ processor for any of reactions (3), (4), or (5) takes about 10 minutes CPU time. This relatively long time of the code generation is determined by a lot of write to and read from disk commands which have to be introduced in order to circumvent limitations of the Fortran compilers concerning possible array sizes. The compilation time of generated routines depends strongly on a compiler used and an optimization option chosen. Typically, for the considered reactions, it takes about one hour to compile all the routines generated for each of the reactions. Most of the time is used for the compilation of the kinematical routines. The execution time of the MC integration with about 2 million calls to the integrand amounts typically to a few hours and, if the MC summing over polarizations is employed, it is dominated by computation of the phase space normalization.

\section{Results}

The numerical results presented in this section have been obtained with the following set of initial physical parameters: the Fermi coupling, fine structure constant in the Thomson limit and strong coupling

$$
G_{\mu}=1.16639 \times 10^{-5} \mathrm{GeV}^{-2}, \quad \alpha_{0}=1 / 137.0359991, \quad \alpha_{s}\left(m_{Z}\right)=0.1176,
$$

the $W$ - and $Z$-boson masses

$$
m_{W}=80.419 \mathrm{GeV}, \quad m_{Z}=91.1882 \mathrm{GeV},
$$

the top quark mass and the heavy external fermion masses of reactions (3), (44) and (5)

$$
m_{t}=174.3 \mathrm{GeV}, \quad m_{b}=4.8 \mathrm{GeV}, \quad m_{c}=1.3 \mathrm{GeV}, \quad m_{\tau}=1.77699 \mathrm{GeV} .
$$

Light fermion masses are neglected except for in the test calculation of the cross section of (4) with the full set of the lowest order Feynman diagrams, mentioned in the previous section. 
The value of the Higgs boson mass is assumed at $m_{H}=130 \mathrm{GeV}$. Widths of unstable particles are calculated to the lowest order of SM resulting in the following values:

$$
\Gamma_{t}=1.53088 \mathrm{GeV}, \quad \Gamma_{W}=2.04764 \mathrm{GeV}, \quad \Gamma_{H}=8.0540 \mathrm{MeV} .
$$

The $Z$ boson width, whose actual value is not relevant in the calculation, is put at its experimental value $\Gamma_{Z}=2.4952 \mathrm{GeV}$. We use the CMS with $\sin ^{2} \theta_{W}$ given by (8) .

We identify jets with their original partons and define the following basic cuts which should allow to detect events with separate jets and/or isolated charged leptons:

- cuts on an angle between a quark and a beam, an angle between two quarks and on a quark energy in reactions (3), (41) and (5):

$$
5^{\circ}<\theta(q, \text { beam })<175^{\circ}, \quad \theta\left(q, q^{\prime}\right)>10^{\circ}, \quad E_{q}>15 \mathrm{GeV},
$$

- cuts on angles between a charged lepton and a beam, a charged lepton and a quark and on energy of the charged lepton, $l=\mu, \tau$, in reactions (41) and (5):

$$
5^{\circ}<\theta(l, \text { beam })<175^{\circ}, \quad \theta(l, q)>10^{\circ}, \quad E_{l}>15 \mathrm{GeV},
$$

- a cut on the missing transverse energy in reaction (4),

$$
\not E^{T}>15 \mathrm{GeV}
$$

- a cut on an angle between the two charged leptons and on the missing transverse energy in reaction (5)

$$
\theta\left(l, l^{\prime}\right)>10^{\circ}, \quad E^{T}>30 \mathrm{GeV} .
$$

The size of the background contributions is illustrated in Table 1, where we give results for the lowest order cross sections of reaction (4) at a few centre of mass system (c.m.s.) energies in the presence of basic cuts (13), (14) and (15). The cross section $\sigma_{\text {all }}$ calculated with the complete set of Feynman diagrams is shown in column 2. The cross section calculated without the gluon exchange diagrams $\sigma_{\text {no QCD }}$ is given in column 3 and the signal cross section $\sigma_{\text {signal }}$ calculated with the 20 signal Feynman diagrams is presented in column 4 . In order to see to what extent the cuts reduce a signal of the associated production and decay of the top quark pair and Higgs boson we give the signal cross section $\sigma_{\text {signal }}^{\text {no }}$ and the signal cross section in the narrow width approximation (NWA) $\sigma_{\mathrm{NWA}}^{\text {no cuts }}$ without cuts [11] in columns 5 and 6, respectively. The numbers in parenthesis show the MC uncertainty of the last decimal. We see that angular and energy cuts (13), (14) and (15) reduce the signal by about 20\%, but they are not very efficient in reducing the background contributions: both $\sigma_{\text {all }}$ and $\sigma_{\text {no QCD }}$ are substantially larger than $\sigma_{\text {signal }}$. In particular, at $\sqrt{s}=500 \mathrm{GeV}$ that is the most realistic collision energy of ILC, the background exceeds the signal by almost an order of magnitude. This is obviously caused by the fact that this collision energy is just above the $t \bar{t} H$ production threshold and the small phase space volume which is then available naturally reduces the signal cross section. Let us also note that, even though the cut on the energy of each quark has been imposed, the QCD background contributions are quite sizeable, as can be seen by comparison of $\sigma_{\text {all }}$ and $\sigma_{\text {no QCD }}$. 


\begin{tabular}{c|c|c|c|c|c}
\hline \hline$\sqrt{s}[\mathrm{GeV}]$ & $\sigma_{\text {all }}[\mathrm{ab}]$ & $\sigma_{\text {no QCD }[\mathrm{ab}]}$ & $\sigma_{\text {signal }}[\mathrm{ab}]$ & $\sigma_{\text {signal }}^{\text {no cuts }}[\mathrm{ab}]$ & $\sigma_{\text {NWA }}^{\text {no cuts }}[\mathrm{ab}]$ \\
\hline 500 & $26.8(4)$ & $7.80(3)$ & $3.095(3)$ & $3.796(3)$ & $3.920(1)$ \\
800 & $100.2(8)$ & $66.8(1)$ & $46.27(2)$ & $58.36(2)$ & $60.03(2)$ \\
1000 & $93.1(3)$ & $61.4(1)$ & $40.18(2)$ & $51.74(2)$ & $52.42(3)$ \\
2000 & $47.4(2)$ & $28.5(1)$ & $15.14(3)$ & $22.14(4)$ & $20.68(3)$
\end{tabular}

Table 1: Cross sections of reaction (4) at different c.m.s. energies with cuts (13), (14) and (15) calculated: with the complete set of Feynman diagrams, $\sigma_{\text {all }}$, without gluon exchange diagrams, $\sigma_{\text {no QCD }}$, and with only the signal diagrams of Fig. 2, $\sigma_{\text {signal }}$. The last two columns show the total signal cross section $\sigma_{\text {signal }}^{\text {no cuts }}$ and the total cross section in NWA $\sigma_{\text {NWA }}^{\text {no cuts }}$ of [11] without cuts. The numbers in parenthesis show the MC uncertainty of the last decimal.

In order to reduce the background let us assume $100 \%$ efficiency of $b$ tagging and define the following invariant mass cuts which should possibly allow to reconstruct $W$ bosons, $t$ quarks and the Higgs boson:

- a cut on the invariant mass of two non $b$ jets in reactions (3) and (4)

$$
60 \mathrm{GeV}<\left[\left(p_{\sim b_{1}}+p_{\sim b_{2}}\right)^{2}\right]^{1 / 2}<90 \mathrm{GeV}
$$

- a cut on the transverse mass of the muon-neutrino system in reaction (44)

$$
\left[m_{\mu}^{2}+2\left(m_{\mu}^{2}+\left|\mathbf{p}_{\mu}^{T}\right|^{2}\right)^{1 / 2}\left|\boldsymbol{p}^{T}\right|-2 \mathbf{p}_{\mu}^{T} \cdot \boldsymbol{p}^{T}\right]^{1 / 2}<90 \mathrm{GeV}
$$

- a cut on the invariant mass of a $b$ jet, $b_{1}$, and two non $b$ jets, $b_{\sim b_{1}}, b_{\sim b_{2}}$, in reactions (3) and (4)

$$
\left|\left[\left(p_{b_{1}}+p_{\sim b_{1}}+p_{\sim b_{2}}\right)^{2}\right]^{1 / 2}-m_{t}\right|<30 \mathrm{GeV}
$$

- a cut on the transverse mass $m_{T}$ of a $b$ quark, $b_{2}$, muon and missing transverse energy system in reaction (4)

$$
m_{t}-30 \mathrm{GeV}<m_{T}<m_{t}+10 \mathrm{GeV}
$$

where

$$
m_{T}^{2}=m^{2}+2\left(m^{2}+\left|\mathbf{p}_{b_{2}}^{T}+\mathbf{p}_{\mu}^{T}\right|^{2}\right)^{1 / 2} \mathbb{E}^{T}-2\left(\mathbf{p}_{b_{2}}^{T}+\mathbf{p}_{\mu}^{T}\right) \cdot \mathbf{p}^{T}
$$

with $m$ being the invariant mass of the $b-\mu$ system given by $m^{2}=\left(p_{b_{2}}+p_{\mu}\right)^{2}$.

- an invariance mass cut on two $b$ jets, $b_{3}$ and $b_{4}$, in reactions (3), (44) and (5)

$$
\left|\left[\left(p_{b_{3}}+p_{b_{4}}\right)^{2}\right]^{1 / 2}-m_{H}\right|<m_{b b}^{\text {cut }}
$$

with $m_{b b}^{\text {cut }}=20 \mathrm{GeV}, 5 \mathrm{GeV}$, or $1 \mathrm{GeV}$. 

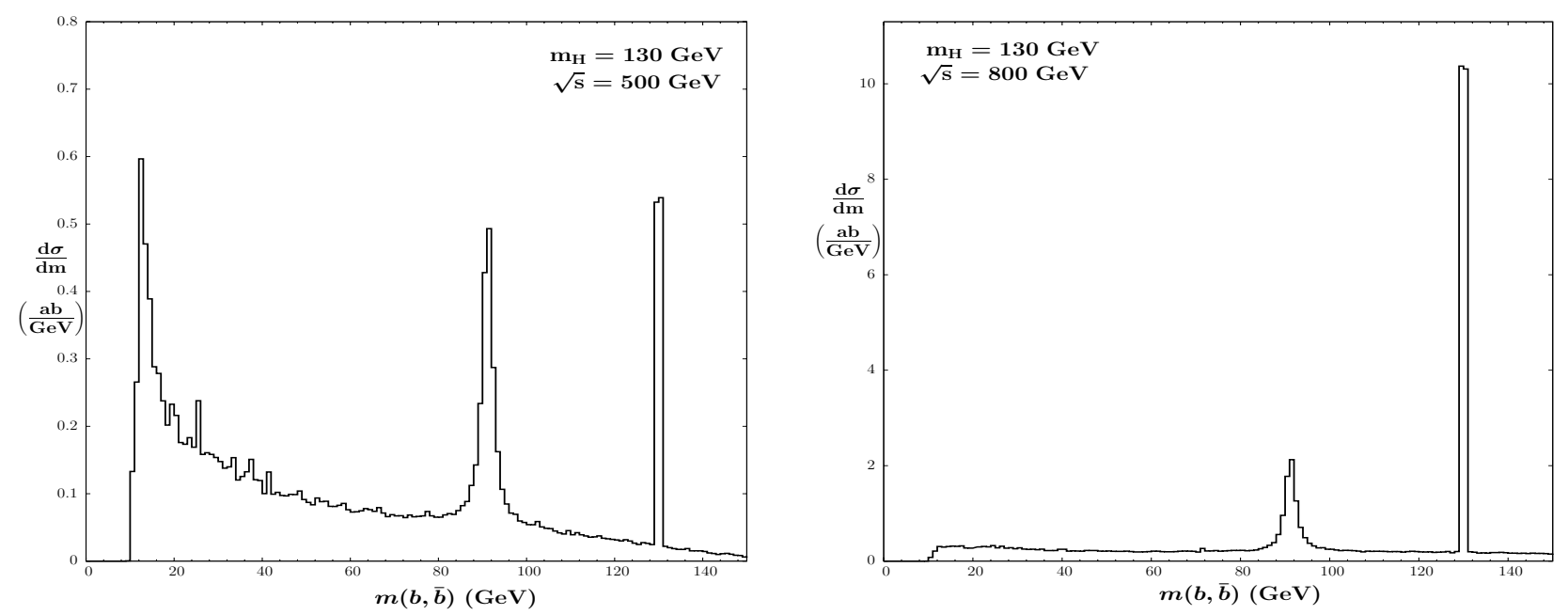

Figure 3: Differential cross section of (4) at $\sqrt{s}=500 \mathrm{GeV}$ (left) $\sqrt{s}=800 \mathrm{GeV}$ (right) as a function of the invariant mass of the two $b$ jets which remain after the other two $b$ jets have been associated with top quarks: one $b$ jet together with the two non $b$ jets have passed a cut (19) and the $\left(b, \mu, \mathbb{E}_{T}\right)$ system has passed a cut (20). The other cuts applied are given by (13), (14), (15), (17) and (18).

In order to justify the actual choice of invariant mass cuts in (20) and (22) let us have a look at plots of some differential cross sections of (4). In Fig. 3, we plot the differential cross section at $\sqrt{s}=500 \mathrm{GeV}$ (left) and $\sqrt{s}=800 \mathrm{GeV}$ (right) as a function of the invariant mass of the two $b$ jets which remain after the other two $b$ jets have passed cuts (19) and (20) in the presence of angular and energy cuts (13), (14) and (15)). How well the top quark mass can be reconstructed from the $\left(b, \mu, \mathbb{E}_{T}\right)$ system is illustrated in Fig. 4, where we plot the differential cross section at $\sqrt{s}=500 \mathrm{GeV}$ (left) and $\sqrt{s}=800 \mathrm{GeV}$ (right) as a function of transverse mass (21) of the $\left(b, \mu, E_{T}\right)$ system that has passed a cut (20). The angular and energy cuts are given by (13), (14) and (15).

In Table 2, we show the lowest order $\sigma_{\text {all }}$ and signal $\sigma_{\text {sig. }}$ cross sections of reactions (3), (4) and (5) at a few c.m.s. energies in the presence of angular, energy and invariant mass cuts.

The actual cutting procedures applied in different channels are the following.

- In the hadronic channel, represented by reaction (3), we first impose angular and energy cuts (13). Then we order the four non $b$ jets in pairs and check if they satisfy $W$ boson identification criteria (17). If so, we combine each pair with a $b$ jet and check if they obey the $t$ identification criteria (19). Finally, we check if the remaining two $b$ jets satisfy the Higgs boson identification cut (22).

- In the semileptonic channel, represented by reaction (44), we impose angular and energy cuts (13), (14) and the missing energy cut (15). Then we check whether the two non $b$ jets satisfy a cut (17), and if the muon and missing energy fulfil a transverse mass cut (18). If so, we combine a $b$ jet with the two non $b$ jets and check if they obey the $t$ identification criteria (19). We select two of the remaining three $b$ jets and check if they 

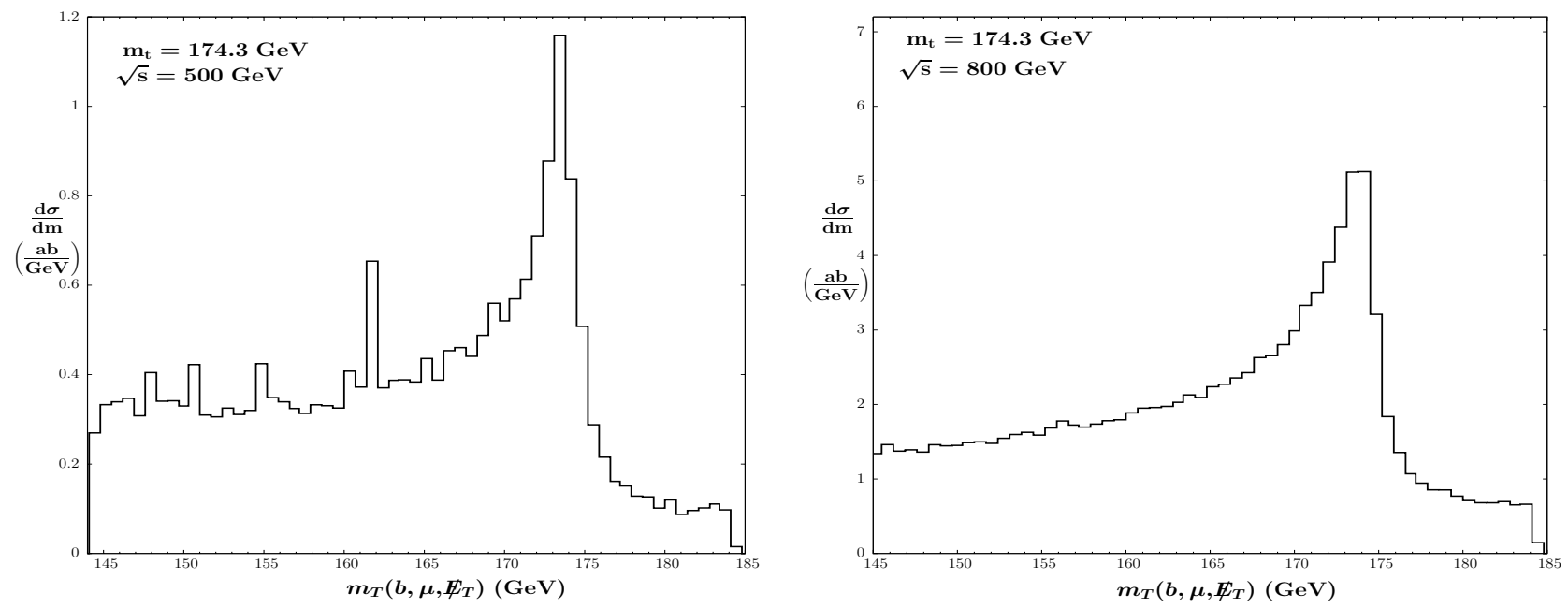

Figure 4: Differential cross section of (4) as a function of the transverse mass, as given by (21), of the $\left(b, \mu, E_{T}\right)$ system that has passed a cut (20) at $\sqrt{s}=500 \mathrm{GeV}$ (left) and $\sqrt{s}=800 \mathrm{GeV}$ (right). The other cuts applied are given by (13), (14), (15), (17) and (18).

fulfil (22). Finally, we impose a transverse mass cut (20) on the other $b$ jet, the muon and missing energy system.

- In the leptonic channel, represented by reaction (5), we impose angular and energy cuts (13), (14) and (16). We select two of the four $b$ jets and check if they satisfy the Higgs boson identification criteria (22).

The cross sections in the first, second and third row for each c.m.s. energy correspond to the Higgs boson invariant mass cut $m_{b b}^{\text {cut }}$ of (22) equal to, respectively, $20 \mathrm{GeV}, 5 \mathrm{GeV}$ and $1 \mathrm{GeV}$. We see that the invariant mass cut on the two $b$ jets which reconstruct the Higgs boson very efficiently reduces the background, practically for all the three detection channels of (2) considered in Table 2. The smaller value of $m_{b b}^{\text {cut }}$ the smaller is the background. The background is relatively the biggest in the leptonic channel, represented by reaction (5), where we have not imposed invariant mass cuts which would allow for reconstruction of $W$ bosons and $t$ quarks.

To which extent an extra cut on the energy of a $b$ quark

$$
E_{b}>40 \mathrm{GeV}, \quad \text { or } \quad E_{b}>45 \mathrm{GeV} .
$$

can reduce the off resonance background contributions is shown in Table 3, where we show the lowest order $\sigma_{\text {all }}$ and signal $\sigma_{\text {sig. }}$ cross section of (44) and (5). The first and second row for each c.m.s. energy correspond to the Higgs boson invariant mass cut $m_{b b}^{\text {cut }}$ of (22) equal to, respectively, $20 \mathrm{GeV}$ and $5 \mathrm{GeV}$. We see that an extra cut (23) on energy of the $b$ quark efficiently reduces the background at $\sqrt{s}=500 \mathrm{GeV}$, practically without altering the signal. However, it reduces both the background and the signal at higher c.m.s. energies. For leptonic reaction (5), where no invariant mass cuts allowing either $W$ bosons or $t$ quarks reconstruction have been imposed, the $b$ quark energy cut (23) helps to reduce the background substantially. 


\begin{tabular}{c|rr|rr|rr}
\hline \hline$\sqrt{s}$ & $e^{+} e^{-} \rightarrow b \bar{b} b \bar{b} u \bar{d} s \bar{c}$ & $e^{+} e^{-} \rightarrow b \bar{b} b \bar{b} u \bar{d} \mu \bar{\nu}_{\mu}$ & $e^{+} e^{-} \rightarrow b \bar{b} b \bar{b} \tau^{+} \nu_{\tau} \mu^{-} \bar{\nu}_{\mu}$ \\
\cline { 2 - 7 }$[\mathrm{GeV}]$ & $\sigma_{\text {all }[\mathrm{ab}]} \sigma_{\text {sig. }}[\mathrm{ab}]$ & $\sigma_{\text {all }}[\mathrm{ab}]$ & $\sigma_{\text {sig. }}[\mathrm{ab}]$ & $\sigma_{\text {all }}[\mathrm{ab}]$ & $\sigma_{\text {sig. }}[\mathrm{ab}]$ \\
\hline \multirow{5}{*}{500} & $13.88(6)$ & $8.70(2)$ & $3.50(2)$ & $2.384(3)$ & $4.03(9)$ & $0.863(2)$ \\
& $10.17(4)$ & $8.66(2)$ & $2.62(1)$ & $2.332(3)$ & $1.89(7)$ & $0.864(2)$ \\
& $9.07(4)$ & $8.65(1)$ & $2.37(1)$ & $2.312(3)$ & $1.09(2)$ & $0.860(1)$ \\
& $167.0(4)$ & $128.4(1)$ & $43.6(1)$ & $33.93(2)$ & $23.28(5)$ & $13.48(1)$ \\
500 & $139.1(3)$ & $128.0(1)$ & $35.8(1)$ & $33.10(2)$ & $16.95(4)$ & $13.47(1)$ \\
& $130.5(2)$ & $127.7(1)$ & $33.4(1)$ & $32.82(2)$ & $14.44(4)$ & $13.46(1)$ \\
& $139.4(3)$ & $109.1(1)$ & $35.3(1)$ & $27.94(1)$ & $21.70(5)$ & $12.09(1)$ \\
& $117.9(5)$ & $109.0(1)$ & $29.5(1)$ & $27.52(1)$ & $15.41(3)$ & $12.10(1)$ \\
& $110.6(2)$ & $108.7(1)$ & $27.8(1)$ & $27.34(1)$ & $13.08(6)$ & $12.06(1)$ \\
& $44.5(2)$ & $36.37(4)$ & $11.4(1)$ & $9.223(6)$ & $9.46(3)$ & $4.95(2)$ \\
& $38.1(1)$ & $36.23(4)$ & $9.76(4)$ & $9.157(6)$ & $6.49(3)$ & $4.97(3)$ \\
& $36.6(1)$ & $36.09(2)$ & $9.25(4)$ & $9.136(6)$ & $5.42(2)$ & $4.97(1)$
\end{tabular}

Table 2: Lowest order, $\sigma_{\text {all }}$, and signal, $\sigma_{\text {sig., }}$ cross sections of reactions (3), (4) and (15). The first, second and third row for each c.m.s. energy correspond to the Higgs boson invariant mass cut $m_{b b}^{\text {cut }}$ of (22) equal to, respectively, $20 \mathrm{GeV}, 5 \mathrm{GeV}$ and $1 \mathrm{GeV}$. Other angular, energy and invariant mass cuts are specified for each reaction in the main text. The numbers in parenthesis show the uncertainty of the last decimal.

\begin{tabular}{|c|c|c|c|c|c|c|c|c|}
\hline \multirow{3}{*}{$\begin{array}{c}\sqrt{s} \\
{[\mathrm{GeV}]}\end{array}$} & \multicolumn{4}{|c|}{$e^{+} e^{-} \rightarrow b \bar{b} b \bar{b} u \bar{d} \mu^{-} \bar{\nu}_{\mu}$} & \multicolumn{4}{|c|}{$e^{+} e^{-} \rightarrow b \bar{b} b \bar{b} \tau^{+} \nu_{\tau} \mu^{-} \bar{\nu}_{\mu}$} \\
\hline & \multicolumn{2}{|c|}{$E_{b}>40 \mathrm{GeV}$} & \multicolumn{2}{|c|}{$E_{b}>45 \mathrm{GeV}$} & \multicolumn{2}{|c|}{$E_{b}>40 \mathrm{GeV}$} & \multicolumn{2}{|c|}{$E_{b}>45 \mathrm{GeV}$} \\
\hline & $\sigma_{\text {all }}[\mathrm{ab}]$ & $\sigma_{\text {sig. }}[a b]$ & $\sigma_{\text {all }}[\mathrm{ab}]$ & $\sigma_{\text {sig. }}[\mathrm{ab}]$ & $\sigma_{\text {all }}[\mathrm{ab}]$ & $\sigma_{\text {sig. }}[\mathrm{ab}]$ & $\sigma_{\text {all }}[\mathrm{ab}]$ & $\sigma_{\text {sig. }}[\mathrm{ab}]$ \\
\hline \multirow{2}{*}{500} & $3.25(1)$ & $2.36(1)$ & $2.93(1)$ & $2.27(1)$ & $1.49(1)$ & $0.850(2)$ & $1.22(1)$ & $0.810(2)$ \\
\hline & $2.57(1)$ & $2.32(1)$ & $2.47(1)$ & $2.22(1)$ & $1.11(1)$ & $0.849(2)$ & $1.02(4)$ & $0.808(2)$ \\
\hline \multirow{2}{*}{800} & $27.9(1)$ & $23.72(3)$ & $24.0(1)$ & $20.58(3)$ & $13.58(4)$ & $9.38(1)$ & $11.51(2)$ & $8.14(1)$ \\
\hline & $24.4(1)$ & $23.07(3)$ & $21.1(1)$ & $20.03(3)$ & $10.99(2)$ & $9.37(1)$ & $9.44(2)$ & $8.13(1)$ \\
\hline \multirow{2}{*}{1000} & $22.7(1)$ & $19.50(2)$ & $20.1(1)$ & $17.49(2)$ & $12.84(3)$ & $8.48(1)$ & $11.18(2)$ & $7.59(1)$ \\
\hline & $20.0(1)$ & $19.20(2)$ & $18.0(1)$ & $17.21(2)$ & $10.07(2)$ & $8.47(1)$ & $8.92(2)$ & $7.59(1)$ \\
\hline \multirow{2}{*}{2000} & $8.59(4)$ & $7.35(1)$ & $8.10(3)$ & $6.95(1)$ & $6.45(2)$ & $4.02(2)$ & $5.93(2)$ & $3.78(2)$ \\
\hline & $7.61(3)$ & $7.28(1)$ & $7.27(6)$ & $6.90(1)$ & $4.84(2)$ & $4.01(2)$ & $4.51(1)$ & $3.84(2)$ \\
\hline
\end{tabular}

Table 3: Lowest order, $\sigma_{\text {all }}$, and signal, $\sigma_{\text {sig. }}$, cross sections of reactions (4) and (5) with an extra cut (23) on the $b$ quark energy. The first and second row for each c.m.s. energy correspond to the Higgs boson invariant mass cut $m_{b b}^{\text {cut }}$ of (22) equal to, respectively, $20 \mathrm{GeV}$ and $5 \mathrm{GeV}$. Other angular, energy and invariant mass cuts are specified for each reaction in the main text. The numbers in parenthesis show the uncertainty of the last decimal. 


\section{Conclusions}

We have calculated the lowest order SM cross sections of reactions (3), (4) and (5) representing the hadronic, semileptonic and leptonic detection channels of the associated production of the top quark pair and Higgs boson at the ILC. In the calculation, we have taken into account complete sets of the lowest order Feynman diagrams, both EW and QCD ones. A comparison of the cross sections with the corresponding signal cross sections of the associated production and decay of the top quark pair and Higgs boson in the presence of angular and energy cuts has shown that the off resonance background is large. Imposing invariant mass cuts, which allow for reconstruction of $W$ bosons, $t$ quarks and the Higgs boson, reduces the background. In particular, a cut (22) on the invariant mass of the two $b$ quark system that reconstructs the Higgs boson is very efficient: the smaller the cut the better is the background reduction. A cut on the energy of the slowest $b$ jet can further reduce the background at $\sqrt{s}=500 \mathrm{GeV}$, but for higher c.m.s. energies it becomes less efficient, as it reduces the signal as well.

Acknowledgements: This work is supported in part by the Polish Ministry of Science under Grant No. N N519 404034 and by European Community's Marie-Curie Research Training Network under contracts MRTN-CT-2006-035482 (FLAVIAnet) and MRTN-CT-2006-035505 (HEPTOOLS).

\section{References}

[1] James Brau, Yasuhiro Okada, Nicholas Walker, et al. [ILC Reference Design Report Volume 1 - Executive Summary], arXiv:0712.1950;

J.A. Aguilar-Saavedra et al. [ECFA/DESY LC Physics Working Group Collaboration], arXiv:hep-ph/0106315;

T. Abe et al., [American Linear Collider Working Group Collaboration], arXiv:hep-ex/0106056;

K. Abe et al. [ACFA Linear Collider Working Group Collaboration], arXiv:hep-ph/0109166.

[2] A. Djouadi, J. Kalinowski, P.M. Zerwas, Mod. Phys. Lett. A7 (1992) 1765;

A. Djouadi, J. Kalinowski, P.M. Zerwas, Z. Phys C54 (1992) 255.

[3] R. Barate et al., Phys. Lett. B565 (2003) 61.

[4] The LEP Collaborations and the LEP electroweak working group, arXiv:hep-ex/0612034 v2, and references therein;

Ch. Parkes, International Conference on High Energy Physics, http://lephiggs.web.cern.ch/, July 2006;

B. Kilminster, arXiv:hep-ex/0611001, to appear in the proceedings of 33rd International Conference on High Energy Physics (ICHEP 06), Moscow, Russia, 26 Jul. - 2 Aug. 2006.

[5] S. Dittmaier, M. Kramer, Y. Liao, M. Spira, P.M. Zerwas, Phys. Lett. B441 (1998) 383;

S. Dittmaier, M. Kramer, Y. Liao, M. Spira, P.M. Zerwas, Phys. Lett. B478 (2000) 247;

S. Dawson, L. Reina, Phys Rev. D57 (1998) 5851;

S. Dawson, L. Reina, Phys Rev. D59 (1999) 054012. 
[6] Yu You et al., Phys. Lett. B571 (2003) 85;

[7] G. Bélanger et al., Phys. Lett. B571 (2003) 163;

A. Denner, S. Dittmaier, M. Roth, M.M. Weber, Phys. Lett. B575 (2003) 290;

A. Denner, S. Dittmaier, M. Roth, M.M. Weber, Nucl. Phys. B680 (2004) 85.

[8] C. Farrell, A.H. Hoang, Phys. Rev. D72 (2005) 014007;

C. Farrell, A.H. Hoang, Phys. Rev. D74 (2006) 014008.

[9] S. Moretti, Phys. Lett. B452 (1999) 338.

[10] C. Schwinn, arXiv:hep-ph/0412028.

[11] K. Kołodziej, S. Szczypiński, Acta Phys. Pol. B38 (2007) 2565-2576.

[12] K. Kołodziej, S. Szczypiński, Acta Phys. Pol. B38 (2007) 3609.

[13] H. Baer, S. Dawson, L. Reina, Phys Rev. D61 (2000) 013002;

A. Juste, G. Merino, arXiv:hep-ph/9910301;

A. Juste, ECONF C0508141:ALCPG0426, 2005, arXiv:hep-ph/0512246;

A. Gay, arXiv:hep-ph/0604034.

[14] K. Kołodziej, "carlomat, a program for automatic computation of multiparticle cross sections", in preparation.

[15] A. Denner, S. Dittmaier, M. Roth, D. Wackeroth, Nucl. Phys. B560 (1999) 33 and Comput. Phys. Commun. 153 (2003) 462.

[16] K. Kołodziej, Comp. Phys. Commun. 151 (2003) 339.

[17] M. Moretti, T. Ohl, J. Reuter, IKDA 2001/06-rev, LC-TOOL-2001-040-rev, hep-ph/0102195-rev;

W. Kilian, T. Ohl, J. Reuter, arXiv:0708.4233. 